\title{
RTI-TRAPS: An Adaptive Vehicle Tracking Methodology for Public Transportation
}

\author{
Bhukya Devi Prasad \\ Council of Scientific and Industrial \\ Research \\ New Delhi, India
}

\author{
Reeta Sony A. L \\ National Law University \\ New Delhi \\ India
}

\begin{abstract}
This paper proposes and implements a low cost vehicle tracking methodology without using the GPS technology. This is of benefit majorly in the public transportation systems. The RTI-TRAPS, which is, "Real Time Informer of Public Transportation Systems" is a methodology that provides the real time information about the vehicle location to the users via SMS. This involves utilizing of already proven communication technology and the integration of human interface into the information flow system structure. The flow system developed is the simplest model and uses the mobile phone with GSM/CDMA capabilities as its important medium for communication. The application is developed using Microsoft Technologies, thus, enabling it to run only on a Windows platform. This paper hence provides with the required architectural details, Hardware Specification and Software Requirements Specification (SRS) details to set up operational RTI-TRAPS. Also a cost comparative analysis is given to show how this methodology stands off in comparison to GPS technology in adaptation.
\end{abstract}

\section{Keywords}

Vehicle Tracking, Public Transportation System, Vehicle Location.

\section{INTRODUCTION}

There are thousands of buses in India which enables thousand and lakhs of people to communicate at various times to various destinations. But, due to lack of real time information about the availability and the timings of these transport vehicles, causing many women, aged people to wait at respective bus stops for really long hours. Normally, static time tables of buses are hardly followed by the buses. Even these time tables are restricted to certain major bus depots. There are many places in India where a bus stand is nothing more than a tree and in such places the only way to know about and travel is to simply wait for the bus and expect when the bus may arrive based on observational experience. Other alternative transport is risky and a lot of valuable time is being wasted everyday simply waiting for buses. This is a challenging problem faced by everyone and calls for technology to solve the problem.

Even in today's technologically advanced world, there are people who can only dream of using technology. The technology developed is not reaching such under-privileged users. In true spirit of engineering, technology shall be used in alleviating the problems faced by rural India. After doing considerable research by interacting with people from different walks of life which include farmers, teachers, students etc in villages and concluded that one of their primary problems is lack of information about transportation i.e. when buses or other vehicles for public transportation arrive and departure. It is quite common in rural locations that the Government buses do not maintain any specific time and people have no way of knowing when the next bus might arrive or even if there is another bus commuting. So they end up waiting for eternity or sometimes take the alternative transportation which are expensive and hazardous too.

The average waiting time for buses in remote village is nearly one hour and this consumes valuable time and energy. This is also a loss to the government which is losing on the revenue generated by transport sector. So, in effect, even though there is infrastructure established, the lack of co-ordination leaves much to be desired. Addressing this problem of the common man by providing him with a very effective way of saving his time and ensuring his safety through technology. Authors believe that this will greatly improve people's life in the country because they will have some reliable information to bank on and make their decisions.

There exist numerous developed systems to track the vehicles [1], tracing its route [2] from the source to its destination. But in all these systems either the operating cost is higher or the set up cost is more which prevents them from adopting it for widespread usages. Thus the objective of this research is to reduce the cost of the tracking system by utilizing the existing infrastructure and making it available to the common man. Authors believed that the best way to reach the masses was by using the mobile networks [3]. Our proposed solution mainly uses the SMS service [4] which is very cheap now a days and even a basic mobile will work this purpose. Hence such a system is definitely needed and proposed system is well suited owing to the fact that it is very cheap and economically viable and at the same time the technology used is already well established and is here to stay. Authors strongly believe proposed solution is both technically and economically feasible and very much implementable in real life. The paper is further organized in the following way: Section 2 introduces methodology, Section 3 explains hardware specification, Section 4 explains software specification, Section5 explains dataflow structure, Section 6 discuss case study, Section 7 why it's a low cost solution and finally, Section 8 presents the conclusions.

\section{METHODOLOGY}

This research mainly originated from interest in providing services to rural India and alleviating some of their problems through technology. Author's has a very strong hold on the ground reality in villages and small towns since author 
belonged to a remote location. So the solution was developed considering all resources available in rural India [5]. The proposed solution is addressing a very common problem with the public bus transportation in India [6] i.e. the lack of a proper real time economic tracking system for the buses. Other alternative transport is risky and a lot of valuable time is being wasted everyday simply waiting for buses. This is a challenging problem faced by everyone and calls for technology to solve the problem.

The basis for proposed system is the use of any mobile phone and seen that almost $95 \%$ of the people who are into some profession and who travel own a mobile. Also the network coverage in India [7] will only improve in the future with more towers and not diminish. Since the solution needs to reach the masses, approach has to be largely based on existing infrastructure. Telecom sector is the most visible sign of India's economic growth today and stands as the most penetrated technology into the Indian masses. One look at the users of over 670 million mobile connections [8], which is roughly one of every two Indians, confirms this; which means almost every family now owns a mobile handset. It is observed that Indian teledensity is showing a growing trend and is expected to increase further. Today the mobile phone is much more than just a communication device and is being used for various service oriented provisions. Thus using their mobiles and the existing wireless and GSM network will be an economically viable solution in order to provide commuters with information about the buses they wish to travel in.

The adopted methodology is a two pronged approach with the User Request Processing and Vehicle Information Processing. In the User Request Processing, User first sends an SMS through their mobile to a RTI-TRAPS number with the information about the area codes of their source location and the final destination. This message is received through a mobile at the server station. The system then processes the request and sends the approximate location of the bus and when it may arrive. This is a real time system; hence the results will be accurate to an acceptable level.

In Vehicle Information Processing, every bus is first allotted a route and a unique mobile number is given to the bus (just a SIM card will do). The route of the bus and its number is then registered and certain codes are allotted to every station which is made known to the driver. The range is from 0 to 9 . Whenever the bus reaches an enroute station, the driver just needs to hold down the corresponding code key on the device in the bus and it automatically generates a message containing the current location and reports back to the server where the server is updated. Each time the user sends a request, data from the server is sent to them through a text message about the location and estimated time of arrival.

To develop this information flow, the needed physical infrastructure thus is mobile handsets with GSM capabilities [9] and a dedicated server station to process the requests. The communication between these infrastructure components is as shown in the figure below, etc.

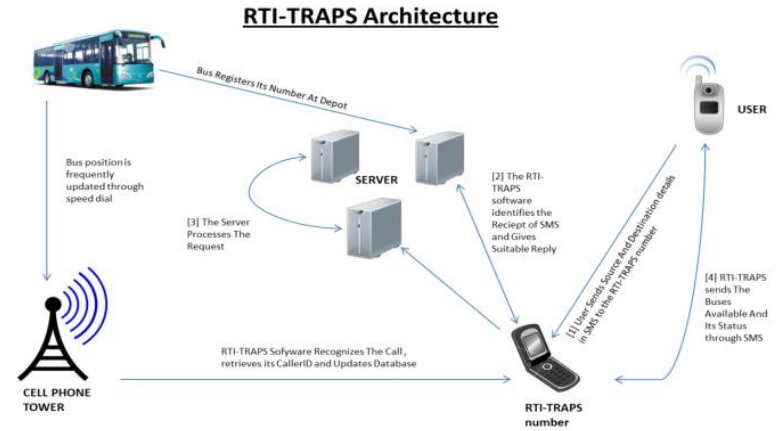

Figure 1: RTI TRAPS Architecture

There is no system implemented in India which dynamically tracks the buses. Hence proposed approach is a novel approach to provide accurate information about buses plying between different places through SMS service. An essential feature of the work is its usability. It requires no technical knowledge on the part of the end users. Proposed solution is depends highly on the network coverage of the Mobile Service Providers [10]. The mobile network coverage is the heart of the proposed system and hence an area with good network coverage [11] is prescribed. The proposed solution and architecture is very cost effective and is based on the existing mobile service providers.

\section{HARDWARE SPECIFICATION}

The major components in this project are the mobile handsets and the servers. Though at the user end mobile handset could be any but at the server end Bluetooth enabled mobile handsets are essential to develop an interface with the Bluetooth enabled PC. Moreover servers are the basic PC which has RTI-TRAPS application installed in to it. In our proposed system, we use following hardware specifications include.

- Intel Pentium IV processor or higher version

- Minimum primary memory of 1GB RAM.

- A hard disk of 80GB HDD is required, which can be further extended.

- $\quad$ Bluetooth enabled mobile phones (5 in number).

- $\quad$ Bluetooth enabled PC.

\section{SOFTWARE SPECIFICATION}

The application is developed using Microsoft Technologies. Though the minimum required environment is an Operating system with Windows XP, Internet explorer (IE 7), Microsoft Visual Studio 2008 with .NET framework v3.5 and SQL Server 2005 but using windows 7 and IE8 will enable the maximum utilization of the features like Super Bar [12] and Accelerators [13] of the application. The application also requires using a few Bluetooth enabled mobiles and PC to function thus software to check each other's compatibility is also essential. 


\section{DATAFLOW STRUCTURE}

The proposed work is intended for use by common man. Anyone with a mobile can access the system for information about the buses. It is assumed that the network coverage in places where the system is being implemented is good and that the users know how to use the SMS service i.e. send an SMS and read an incoming message. The users for this system would be bus commuters both in urban and rural sectors like: Students, Teachers, vendors, Laborers and daily commuters.

In effect any person wanting to know information about the bus he needs to catch without waiting and irrespective of his location. The data set also called data cloud includes Bus Registration ID, Update information, SMS by the User. The complete processing of the system about how the data flows is shown in figure 2. Each Bus registers itself with the RTITRAPS Software and updates itself with it frequently when it is enroute and the database is updated. The algorithm for bus registration and location updation is shown in figure 3. The Input to system from user perspective is an SMS with source and destination as its content. The System process the data retrieves information from the database about the buses that matches the criteria and then sends best three buses information in the form of SMS to the user as a reply. The algorithm processing the User request is shown in figure 4.

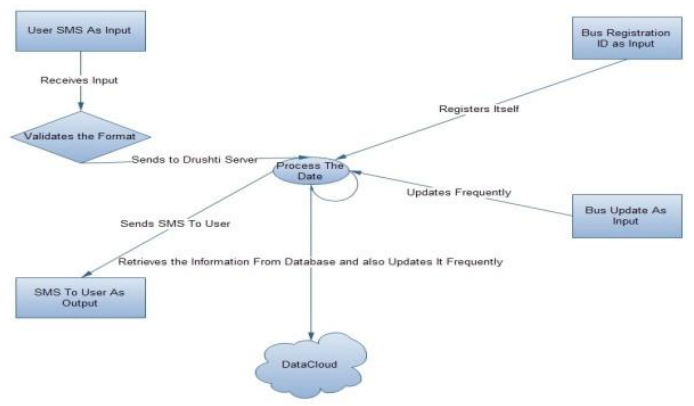

Figure 2: Data Flow Diagram

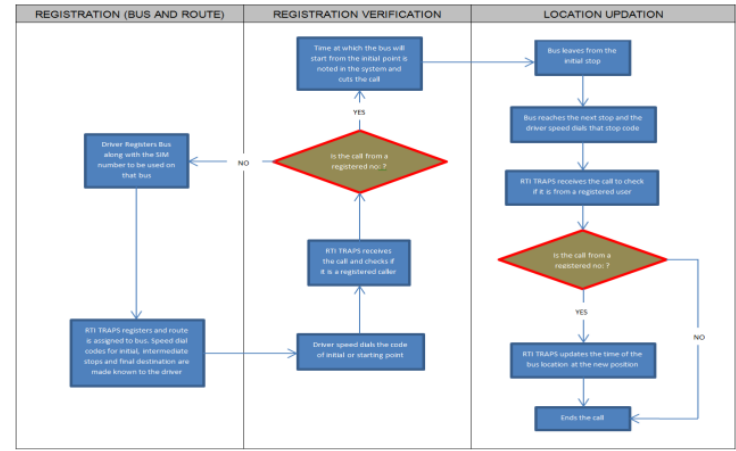

Figure 3: Algorithm to register bus with its route and update its location enroute with RTI TRAPS

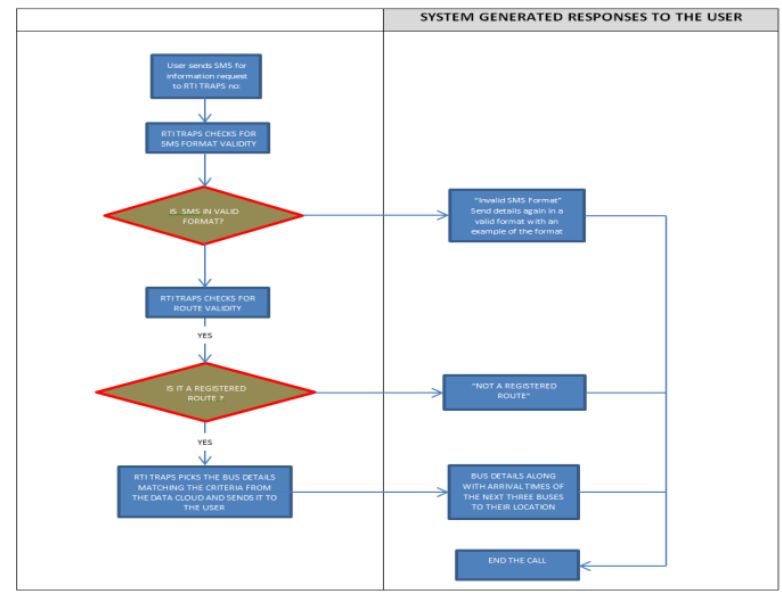

Figure 4: Algorithm to process the User Request with RTI TRAPS

\section{CASE STUDY}

Route Registration:

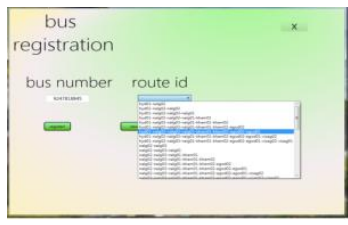

Figure 5a

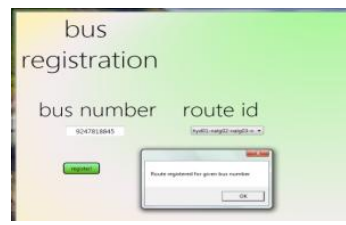

Figure $5 b$
The user interacts with the system via SMS. The user sends an SMS which includes the source and destination. This is received by the system and processed. Then the appropriate response is sent. It is assumed that the network coverage in places where the system is being implemented is good and that the users know how to use the SMS service i.e. send an SMS and read an incoming message.

There are different inputs given to the system through end user in different scenarios and the system responds accordingly. The scenarios are:

- Updation of the current location of the buses through speed dialing. Each time the bus reaches a new location it has to let the system know of the change by dialing a number via speed dial feature. The mobile dialed is one of the mobiles connected to the system. An interface extracts the incoming number and gives it as input to the system. The resulting action is the updation of the database with the location which is in turn obtained based on some codes from the database.

- User request processing. The input is the proper formatted SMS from the user. The SMS is of the form src-dest. The output is the sending of best three buses to the user via an SMS.

- Bus registrations. Here the input is the complete details about a bus which include the route taken by the bus, the mobile number associated with the bus and the approximate time it takes between stops. 
The driver feeds his mobile number in to the 'bus number' text box and then selects the route from the dropdown menu that is available in the 'route id' as shown in figure 5a. After feeding the mobile number and selecting the route in their respective locations one has to register the two. Upon registration a confirmation status appears in the dialogue box as shown in figure $5 b$.

User's Request and RTI TRAPS Server response to the user: User sends the request in the "Source destination" format to the RTI TRAPS number. For illustration purpose two feeds are shown in the table below. Figure 6a is the feed and its related response to the feed where the destination is the intermediary or falls in the prescribed route registered whereas Figure $6 \mathrm{~b}$ is the feed whose destination is not under the prescribed route registered and its related response. The RTI TRAPS response to the former feed is to give the latest three available options and the expected timeframe for each of those options whereas the response to the later feed is to reply "no bus".

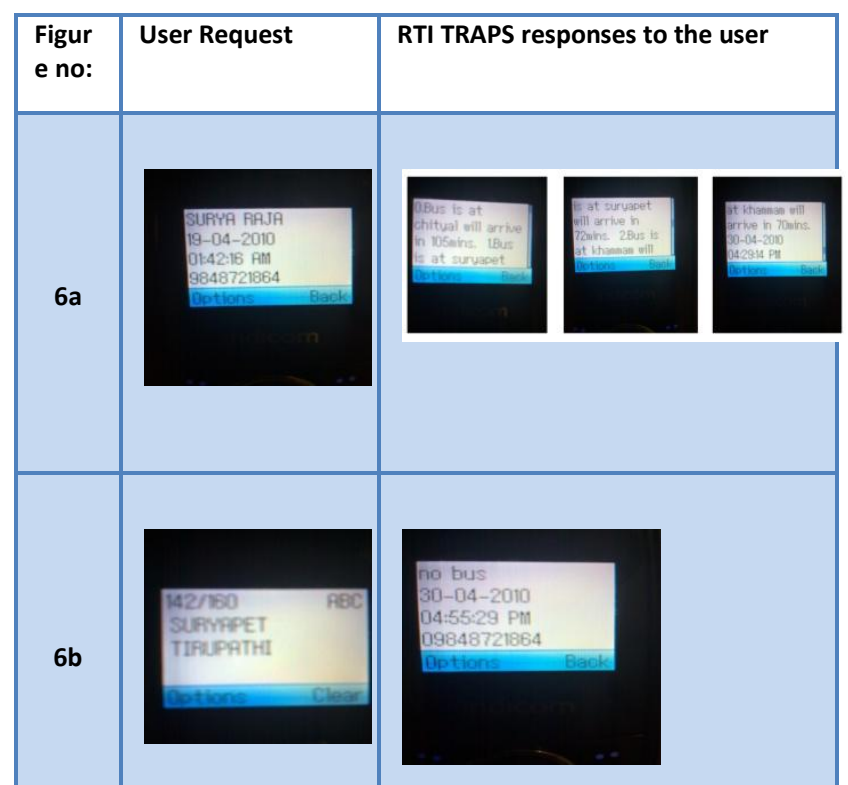

Table 1: Illustrations of User's Request and RTI TRAPS response to the user

7. WHY IS IT A LOW COST SOLUTION?

The proposed methodology has potential to drastically cut down the tracking costs by $60-80 \%$. To set up an operational tracking system for public transportation would involve incurring of three factor costs. One is in incorporating a tracking device on the vehicle, second is in setting up a server station and the third is in sending SMS's to communicate with the user. To get an overview about the estimate these costs are shown for a Metropolitan transport corporation (MTC) that operates a fleet strength of 3260 buses per day with atleast 8 depot clusters.

\section{Fleet strength of MTC $=3260$ buses per day}

If each tracking device costs ' $\mathrm{T}$ ' then total cost of incorporating tracking devices into MTC $=3260 * \mathrm{~T}$
2. Number of Depot clusters $=8$

Each server costs Rs 40000

The total cost for setting up server stations by MTC would be $8 * 40000=$ Rs 320000

3. Number of commuters who take the bus: 6 million

Estimated number of messages to be processed: 4 million

Cost per message via a web-service: 6 ps.

Thus Cost incurred on messages (X): Rs 2.4 lakh per day.

Total cost $=1+2+3=3260 * \mathrm{~T}+320000+$ 240000

$$
=3260 * \mathrm{~T}+560000
$$

It can thus be observed that the cost of the tracking unit plays a very crucial role in adoption for public transportation systems. Currently cost of a GPS tracking unit is at least 5 times the cost of the basic mobile handset. Thus adoption by using a GPS system would be at least 4 times more expensive than by using a basic mobile handset. This adoption cost can be further bought down to bare minimum by trying to utilize the mobile handsets of the drivers who are using it for their personal use. But this is possible only in the country situations like India where the mobile penetration into the masses is high. The cost comparison in three specified adoption scenarios is shown in the table 2 :

\begin{tabular}{|c|c|c|c|}
\hline \multirow{2}{*}{} & \multicolumn{3}{|c|}{ Adoption Scenarios } \\
\cline { 2 - 4 } & GPS & Basic Mobile (new) & $\begin{array}{c}\text { Driver's } \\
\text { Mobile }\end{array}$ \\
\hline $\begin{array}{l}\text { Cost of } \\
\text { each unit } \\
\text { in } \\
\text { Rupees }\end{array}$ & TGPS =5000 & TBM =1000 & TDM =0 \\
\hline $\begin{array}{c}\text { Total } \\
\text { cost } \\
(3260 * \mathrm{~T} \\
+\end{array}$ & $\begin{array}{c}3260 * 5000+ \\
560000= \\
\text { Rs.16.86 } \\
\text { million }\end{array}$ & $\begin{array}{c}3260 * 1000+560000 \\
=\text { Rs 3.82 million }\end{array}$ & $\begin{array}{c}3260 * 0+ \\
560000= \\
\text { Rs } 0.56 \\
\text { million }\end{array}$ \\
\hline
\end{tabular}

Table 2: Adoption Scenarios

\section{CONCLUSION}

This paper proposes using the established mobile infrastructure as a low cost tracking mechanism without the need of a GPS device on the vehicle's used for mass transportation systems or logistics where the rendered services include giving the locational details in real time. The potential advantages in adopting this mechanism are: (a) It uses the existing infrastructure eliminating the need for a separate infrastructure establishments; (b) It can be accessed from anywhere that has good network coverage; (c) It is very simple at the user/customer end as they do not require any additional component/equipment or involves usage of a software application which may or may not be adaptable with the device; (d) Alerts only when the customer wishes to be 
informed; (e) Cost effective not only to the user but also to the service providers and developers.

On a future note this mode of operation can further be developed by using embedded systems which would cost less than a mobile. Since we are utilizing the existing networks, it all boils down to only a couple of things. The use of an embedded system would further add to the system. This would greatly reduce the burden on the conductor. Hence such a system is definitely needed and our system is well suited owing to the fact that it is very cheap and economically viable and at the same time the technology used is already well established and is here to stay.

\section{ACKNOWLEDGMENTS}

The support of Mr. Karthik Reddy,Mr. Sai Kalyan Reddy and others during my doctoral research program at Osmania University, Hyderabad is being acknowledged.

\section{REFERENCES}

[1] Hwajeong Lee, Daehwan Kim, Daijin Kim, Sung Yang Bang , "REAL-Time Automatic Vehicle Management System Using Vehicle Tracking And Car Plate Number Identification ", IEEE International Conference On Multimedia And Expo, 2003.

[2] Santos, M. ; Pereira, R.L. ; Leal, A.B, "GBUS - Route GeoTracer", First International Workshop on Vehicular Traffic Management for Smart Cities, 2012.

[3] Huber, J.F. "Mobile next-generation networks", IEEE MultiMedia,2004

[4] Lim Tai Ching ; Garg, H.K , "Designing SMS applications for public transport service system in Singapore", The 4th International Conference on Frontier of Computer Science and Technology, 2009
[5] Shenggen Fan ,Peter Hazell, Sukhadeo Thorat, "Government Spending, Growth and Poverty in Rural India", American Journal of Agricultural Economics, 2000.

[6] SK Singh ,"Review of urban transportation in India", Journal of Public Transportation, 2005.

[7] Mayank Aggarwal and Vijit Gupta , "Comparative study of telecom service providers in India", IEEE Systems and Information Engineering Design Symposium, 2009.

[8] Jean Paul Simon "The ICT Landscape in BRICS Countries: Brazil, India, China", JRC scientific and Technical reports, 2011.

[9] George Neonakis Aggélou and Rahim Tafazolli, "On the relaying capability of next-generation GSM cellular networks", IEEE Personal Communications, 2011

[10] Aggarwal, M. ; Gupta, V , "Comparative study of telecom service providers in India", Systems and Information Engineering Design Symposium, 2009.

[11] C Hsin, M Liu , “ Network coverage using low dutycycled sensors: random \& coordinated sleep algorithms", third international symposium on Information processing in sensor networks, 2004

[12] Li Ping , Cai Changyu,, Zhu Ling, "Research on Application of Ajax and Silverlight Technology in WebGIS", 1st International Conference on Information Science and Engineering (ICISE), 2009

[13] Tim Cerling, Jeff Buller, Chuck Enstall, Richard Ruiz, "Microsoft Virtualization Tools and Resources", Wiley Publishing, 